\title{
A Metastable Model for Quantum Superposition
}

\author{
Abraham Boyarsky ${ }^{1, *}$, Pawel Góra ${ }^{1}$, Zhenyang Li $^{2}$ \\ ${ }^{1}$ Department of Mathematics and Statistics, Concordia University, 1455 de Maisonneuve Blvd. West, Montreal, Quebec H3G 1M8, \\ Canada. \\ ${ }^{2}$ Department of Mathematics, Honghe University, Mengzi, Yunnan 661100, China.
}

How to cite this paper: Abraham Boyarsky, Paweł Góra, Zhenyang Li. (2020) A Metastable Model for Quantum Superposition. Journal of Applied Mathematics and Computation, 4(4), 224-229.

DOI: $10.26855 /$ jamc.2020.12.013

Received: October 22, 2020

Accepted: November 25, 2020

Published: December 15, 2020

${ }^{*}$ Corresponding author: Abraham Boyarsky, Department of Mathematics and Statistics, Concordia University, 1455 de Maisonneuve Blvd. West, Montreal, Quebec H3G 1M8, Canada.

Email: abraham.boyarsky@concordia.ca

\begin{abstract}
Quantum superposition is often observed as a two peaked probability density function. From this the conclusion is drawn that the quantum particle exists in two places at once. In this note, we present a metastable dynamical systems model which displays a two peaked probability density function but is generated by a process that is in one place at a time. Thus, whenever a measurement is taken, the particle is found where it happens to be at that moment.
\end{abstract}

\section{Keywords}

Quantum superposition, metastable dynamical system, density function, extreme points

\section{Introduction}

Quantum superposition posits that quantum particles have no fixed location. Their existence is spread probabilistically across the domain of a wave function. However, when a macro measurement is made there is an instantaneous and dramatic change: all quantum effects vanish and real properties immediately appear. Quantum theory does not explain why and what happens as probability turns into certainty. This transition is called collapse of the wave function and the mysterious effect is referred to as the measurement problem.

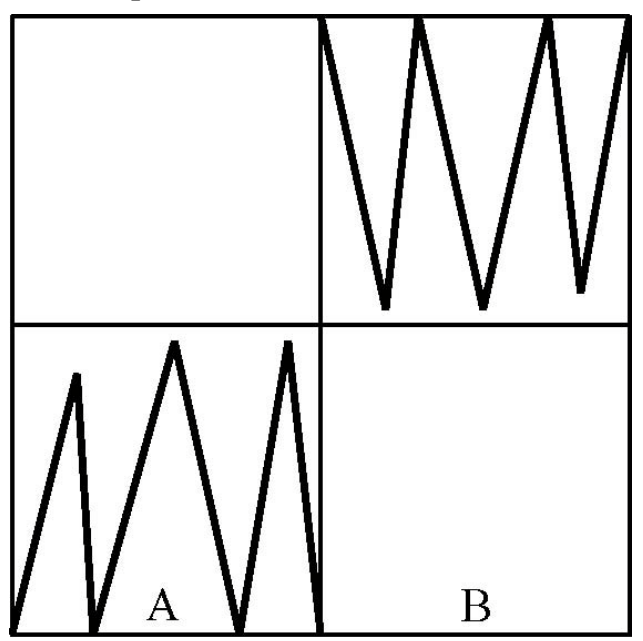

Figure 1. Map T. 
A common way of portraying the measurement problem is to display the probability density function (pdf) of finding a quantum particle. In the quantum case it is a pdf $\mathrm{f}$ with two narrow peaks [1, p. 31]. When a measurement is made one or other of the two peaks vanishes and the quantum particle is localized to the remaining single peak region of the state space. There are various explanations for this effect: for example, decoherence and continuous spontaneous localization, but neither are completely satisfactory. In this note, we present a deterministic metastable system to model the underlying dynamics of quantum superposition. We start with a map T which is defined on an interval I, where I = A U B, and A and $\mathrm{B}$ are disjoint sets. T has two mutually singular ergodic absolutely continuous invariant measures (acim), $\mu_{\mathrm{A}}$ and $\mu_{\mathrm{B}}$, supported on $A$ and $B$, respectively, with probability density functions (pdf) $f_{A}$ and $f_{B}$. Figure 1 shows a map T and Figure 2 displays disjoint probability densities. (Realistic maps and densities are shown in Figures 4 and 5 ). To model the motion of a quantum particle which can be found in either $\mathrm{A}$ or $\mathrm{B}$, we define a metastable map $\mathrm{T}_{\varepsilon}$, (Figure 3) which is an $\varepsilon$ deterministic perturbation of $\mathrm{T} . \mathrm{T}_{\varepsilon}$ causes the sets $\mathrm{A}$ and $\mathrm{B}$ to communicate and has a single ergodic acim, $\mu_{\varepsilon}$, on A U B.

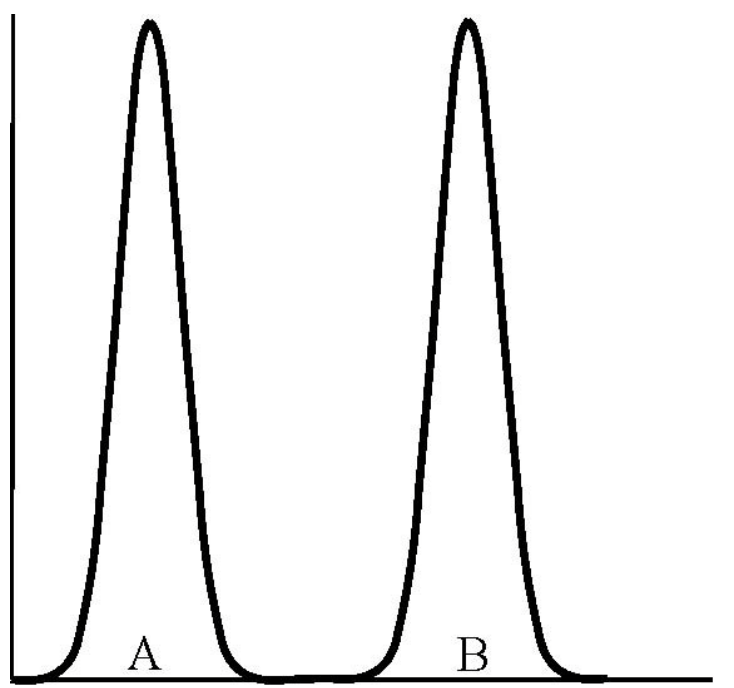

Figure 2. Probability density function with two separated peaks.

In this note, we present a deterministic metastable system to model the underlying dynamics of quantum superposition. We start with a map $\mathrm{T}$ which is definedon an interval $\mathrm{I}$, where $\mathrm{I}=\mathrm{A} \mathrm{U} \mathrm{B}$, and $\mathrm{A}$ and $\mathrm{B}$ are disjoint sets. T has two mutually singular ergodic absolutely continuous invariant measures (acim), $\mu_{A}$ and $\mu_{B}$, supported on A and B, respectively, with probability density functions (pdf) $\mathrm{f}_{\mathrm{A}}$ and $\mathrm{f}_{\mathrm{B}}$. Figure 1 shows a map $\mathrm{T}$ and Figure 2 displays disjoint probability densities. To model the motion of a quantum particle which can be found in either $\mathrm{A}$ or $\mathrm{B}$, we define a metastable map $\mathrm{T}_{\varepsilon}$, (Figure 3 ) which is an $\varepsilon$-deterministic perturbation of $\mathrm{T}$. $\mathrm{T}_{\varepsilon}$ causes the sets $\mathrm{A}$ and $\mathrm{B}$ to communicate and has a single ergodic acim, $\mu_{\varepsilon}$, on A U B.

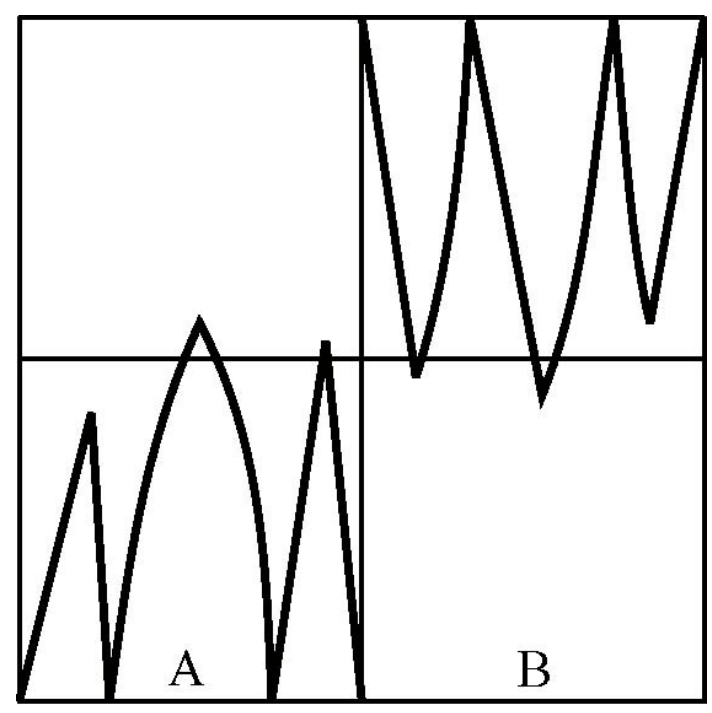

Figure 3. Map $\mathrm{T}_{\varepsilon}$ 
In Section 2, a metastable system is defined and the communication between A and B via escape holes is explained. It is shown that $\mu_{\varepsilon}$ has a pdf $f_{\varepsilon}$ which converges to a convex combination of $f_{A}$ and $f_{B}$ as $\varepsilon \rightarrow 0$. The set of densities $D$ that can be attained by the metastable system depends on the limit of the ratio of hole sizes in A and B and includes all possible convex combinations of $f_{A}$ and $f_{B}$. In Section 3 , we show that the extreme points of $D$ are $f_{A}$ and $f_{B}$. In Section 4 , we construct a metastable map with peaked densities. The main idea of this note is to identify the measurement of a quantum system as an extreme event in a metastable system.

\section{Metastable Dynamical System}

We assume the map $\mathrm{T}: \mathrm{A} \mathrm{U} \mathrm{B} \rightarrow \mathrm{A} \mathrm{U} B$ is a piecewise $\mathrm{C}^{2}$, uniformly expanding on a partition set $\mathrm{C}_{0}=\left\{\mathrm{c}_{0,0}<\mathrm{c}_{1,0}\right.$ $\left.<\cdots<\mathrm{c}_{\mathrm{n}, 0}\right\}$ which is called a critical set. $\mathrm{T}$ has two invariant sets, $\mathrm{A}$ and $\mathrm{B}$. The point $\mathrm{b}=\mathrm{A} \cap \mathrm{B}$ is called a boundary point and the points in $\mathrm{H}_{0}:=\mathrm{T}^{-1}(\{\mathrm{~b}\}) \backslash\{\mathrm{b}\}$ are called infinitesimal holes. The metastable perturbed system $\mathrm{T}_{\varepsilon}$ : $\mathrm{A} U \mathrm{~B}$ $\rightarrow$ A U B of T, which is also piecewise $C^{2}$, has partition set $C_{\varepsilon}=\left\{c_{0, \varepsilon}<c_{1, \varepsilon}<\cdots<c_{n, \varepsilon}\right\}$. The following properties are assumed:

(I1) Unique acim on the initial invariant set:

$\left.T\right|_{A}\left(\left.T\right|_{B}\right)$ has only one acim $\mu_{A}\left(\mu_{B}\right)$ whose density is denoted by $f_{A}\left(f_{B}\right)$.

(I2) No return of the critical set to the infinitesimal holes:

for every $\mathrm{k}>0, \mathrm{~T}^{\mathrm{k}}\left(\mathrm{C}_{0}\right) \cap \mathrm{H}_{0}=$ empty set.

(I3) Positive acims at infinitesimal holes:

$f_{A}$ is positive at each of the points in $H_{0} \cap A$, and $f_{B}$ is positive at each of the points in $H_{0} \cap B$.

(I4) Restriction on periodic critical points. One of the following holds:

(I4a) $\inf _{\mathrm{A} \cap \mathrm{B} \backslash\{\mathrm{C} 0\}}\left|\mathrm{T}^{\prime}(\mathrm{x})\right|>2$;

(I4b) $\mathrm{T}$ has no periodic critical points, except possibly a fixed point at 0 or 1 .

(P1) Unique acim: for each $\varepsilon>0, \mathrm{~T}_{\varepsilon}$ has only one acim $\mu_{\varepsilon}$ with density $\mathrm{f}_{\varepsilon}$.

(P2) Boundary condition:

the boundary point does not move, and no holes are created near the boundary. To be precise:

(P2a) if $\mathrm{b}$ is not in $\mathrm{C}_{0}$, then necessarily $\mathrm{T}(\mathrm{b})=\mathrm{b}$, and we assume further that for all $\varepsilon>0, \mathrm{~T}_{\varepsilon}(\mathrm{b})=\mathrm{b}$;

(P2b) if $\mathrm{b}$ is in $\mathrm{C}_{0}$, we assume that $\mathrm{T}\left(\mathrm{b}_{-}\right)<\mathrm{b}<\mathrm{T}\left(\mathrm{b}_{+}\right)$and also that $\mathrm{b}$ inC $\mathrm{C}_{\varepsilon}$ for all $\varepsilon>0$.

Then, we have the following properties:

$\mathrm{T}_{\varepsilon}$ has only one ergodic acim $\mu_{\varepsilon}$ on $\mathrm{A} U \mathrm{~B}$ with pdf $\mathrm{f}_{\varepsilon}$. We have two holes

$\mathrm{H}_{\mathrm{A}, \varepsilon}:=\mathrm{A} \cap \mathrm{T}_{\varepsilon}^{-1}(\mathrm{~B})$ and $\mathrm{H}_{\mathrm{B}, \varepsilon}:=\mathrm{B} \cap \mathrm{T}_{\varepsilon}{ }^{-1}(\mathrm{~A})$ in $\mathrm{A}$ and $\mathrm{B}$, respectively, through which the orbit of $\mathrm{T}_{\varepsilon}$ escapes from one set to the other set. Once an orbit enters a hole, it leaves one of the invariant sets for $\mathrm{T}$ and continues in the other. As $\varepsilon \longrightarrow$ 0 , the holes converge to the place from which they arise, which are called infinitesimal holes. By the conditions above, both $\mu_{\mathrm{A}}\left(\mathrm{H}_{\mathrm{A}, \varepsilon}\right)$ and $\mu_{\mathrm{B}}\left(\mathrm{H}_{\mathrm{B}, \varepsilon}\right)$ converge to 0 as $\varepsilon \rightarrow 0$.

Theorem 1. [2] Consider the family of perturbations $\left\{\mathrm{T}_{\varepsilon}: \varepsilon>0\right\}$ of $\mathrm{T}$ under the assumptions (I1-I4) and (P1-P2) stated in Section 2.1 of [2]. Suppose that l.h.r. $=\lim _{\varepsilon \rightarrow 0} \mu_{\mathrm{B}}\left(\mathrm{H}_{\mathrm{B}, \varepsilon}\right) / \mu_{\mathrm{A}}\left(\mathrm{H}_{\mathrm{A}, \varepsilon}\right)$ exists. Then

as $\varepsilon \rightarrow 0$, where $\alpha /(1-\alpha)=1$.h.r.

$$
\mathrm{f}_{\varepsilon} \rightarrow \alpha \mathrm{f}_{\mathrm{A}}+(1-\alpha) \mathrm{f}_{\mathrm{B}}
$$

\section{Extreme Metastable Systems}

The extreme values of all l.h.r. are 0 and $\infty$. Hence the extreme points of the setof densities $\left\{f_{\varepsilon}: \varepsilon>0\right\}$ occur if

l.h.r. $=0$, then $\mathrm{f}_{\varepsilon} \rightarrow \mathrm{f}_{\mathrm{B}}$ in $\mathrm{L}^{1}$ as $\varepsilon \rightarrow 0$ or if 1.h.r. $=1$, then $\mathrm{f}_{\varepsilon} \rightarrow \mathrm{f}_{\mathrm{A}}$ in $\mathrm{L}^{1}$ as $\varepsilon \rightarrow 0$. Thus, $\mathrm{f}_{\mathrm{A}}$ and $\mathrm{f}_{\mathrm{B}}$ are the extreme points of $\left\{\mathrm{f}_{\varepsilon}: \varepsilon>0\right\}$ and correspond to what happens once a measurement of a quantum system is made. We have modeled the act of measurement by an extreme state for the metastable system and have shown that this forces the system to display either $\mathrm{f}_{\mathrm{A}}$ od $\mathrm{f}_{\mathrm{B}}$. The macro measurement effectively shuts the escape holes. The measurement process has caused a collapse of the wave function: a two peaked density function has become a one peaked density, depending on which almost invariant set the mouse was in at the time the measurement was taken.

\section{Construction of the map $T$}

In this section we construct an example of a metastable map satisfying the conditions of Section 2 and preserving a density which is a rough approximation of the density $\mathrm{f}$ of Figure 2. A specific example is presented at the end of the section. We start by constructing a map g: $\mathrm{B} \rightarrow \mathrm{B}, \mathrm{B}=[0,1]$, preserving a piecewise constant approximation of an arbitrary pdf $\mathrm{f}_{0}$. Let $\mathrm{P}=\left\{\mathrm{I}_{\mathrm{i}}: 1 \leq \mathrm{i} \leq \mathrm{N}\right\}$ be a partition $\mathrm{B}$ into $\mathrm{N}$ equal subintervals. Let us define the vector $\mathrm{v}=\left[\mathrm{v}_{1}, \mathrm{v}_{2}, \ldots, \mathrm{v}_{\mathrm{N}}\right]$, wherev $_{i}=\int_{\mathrm{Ii}} \mathrm{f}_{0} \mathrm{dm}$. Then, the function

$$
\mathrm{f}_{0 \alpha}(\mathrm{x})=\sum_{\{\mathrm{i}=1, \ldots, \mathrm{N}\}} \mathrm{N} \mathrm{v}_{\mathrm{i}} \chi_{\mathrm{li}}(\mathrm{x})
$$


is a piecewise constant approximation of $\mathrm{f}_{0}$.

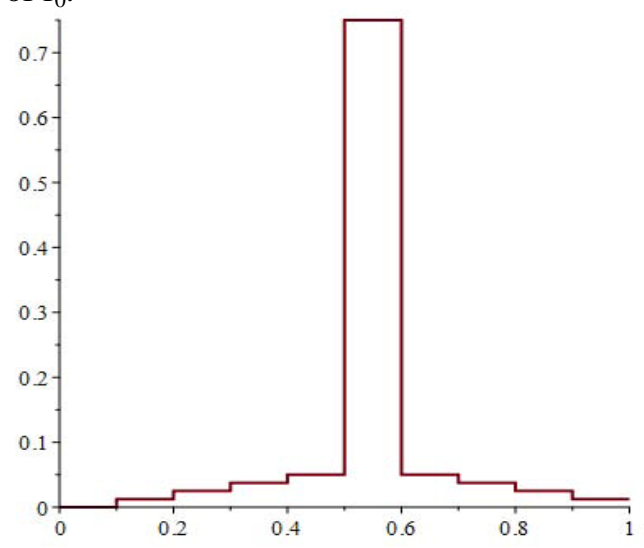

Figure 4. Density $f_{B}=f_{0 \alpha}$ invariant for map g.

Let us choose $0<\beta_{\mathrm{i}}<1$ and set $\alpha_{\mathrm{i}}=\mathrm{v}_{\mathrm{i}}\left(1-\beta_{\mathrm{i}}\right)$, $\mathrm{i}=1, \ldots, \mathrm{N}$. Let

$$
M=\left[\begin{array}{ccccc}
\beta_{1} & 0 & \ldots & 0 & 0 \\
0 & \beta_{2} & 0 & \ldots & 0 \\
\vdots & \vdots & \ddots & \vdots & \vdots \\
0 & 0 & \ldots & \beta_{N-1} & 0 \\
0 & 0 & \ldots & 0 & \beta_{N}
\end{array}\right]+\frac{1}{\sum_{i=1}^{N} \alpha_{i}}\left[\begin{array}{c}
1-\beta_{1} \\
1-\beta_{2} \\
\vdots \\
1-\beta_{N-1} \\
1-\beta_{N}
\end{array}\right]\left[\alpha_{1}, \alpha_{2}, \ldots, \alpha_{N}\right]
$$

The matrix $\mathrm{M}$ is row-stochastic and the vector $\mathrm{v}$ is its left invariant vector. This fact was used in [3] to introduce another method of constructing a piecewise linear map g preserving the pdf $\mathrm{f}_{0 \alpha}$. We use the matrix $\mathrm{M}=\left[\mathrm{m}_{\mathrm{i}, \mathrm{j}}\right]_{1 \leq \mathrm{i}, \mathrm{j} \leq \mathrm{N}}$ to define g which transforms linearly the subinterval of length $m_{i j} / N$ of $I_{i}$ increasingly onto the interval $I_{j}$ if $i$ is odd and decreasingly onto the interval $\mathrm{I}_{\mathrm{N}-\mathrm{j}+1}$ if $\mathrm{i}$ is even. For more details and a general theory of such semi-Markov maps see [4]. We make the construction slightly more complicated than usual to allow the map g to be continuous. Map g is piecewise expanding and piecewise onto so pdf $\mathrm{f}_{0 \alpha}$ is the unique g-invariant pdf (for the general theory of piecewise expanding maps see [5] or [6]).

Now we can define the map $\mathrm{T}:[-1,1] \rightarrow[-1,1]$ by

$$
T(x)=\left\{\begin{array}{c}
g(x), \text { for } x \text { in }[0,1] ; \\
-g(-x), \text { for } x \text { in }[-1,0] .
\end{array}\right.
$$

The map $\mathrm{T}$ has two ergodic components and preserves two invariant pdfs with disjoint supports: $\mathrm{fB}(\mathrm{x})=\mathrm{f} 0 \alpha(\mathrm{x})$ on $\mathrm{B}=$ $[0,1]$ and $\mathrm{fA}(\mathrm{x})=\mathrm{f} 0 \alpha(\mathrm{x})$ on $\mathrm{A}=[-1,0]$. The point $\{0\}=\mathrm{A} \cap \mathrm{B}$ is the fixed point of $\mathrm{T}$. We now open small gates around -1 and 1 : the new map $\mathrm{T}_{\varepsilon}$ is equal to $\mathrm{T}$ on $[-1+\varepsilon, 1-\varepsilon]$ and we have $\mathrm{T}_{\varepsilon}([-1,-1+\varepsilon])=[0, \alpha(\varepsilon)]$ and $\mathrm{T}_{\varepsilon}([1-\varepsilon, 1])=$ $[-\alpha(\varepsilon), 0]$. The map $T_{\varepsilon}$ is metastable and preserves a unique density $f_{\varepsilon}$ which is very close $(1 / 2) f A+(1 / 2) f B$ since $T_{\varepsilon}$ is symmetric and both $\mathrm{T}$ and $\mathrm{T}_{\varepsilon}$ are piecewise expanding [5].

For a specific example we fix $N=10$. We consider density $f B=f 0, \alpha$ corresponding to the vector $v=(1 / 80)[0,1,2,3$, 4, 60, 4, 3, 2, 1] (Figure 4). The semi-Markov map g constructed as above is shown in Figure 5 . The maps $\mathrm{T}_{\text {and }} \mathrm{T}_{\varepsilon}$ are shown in Figures 6 and 7.

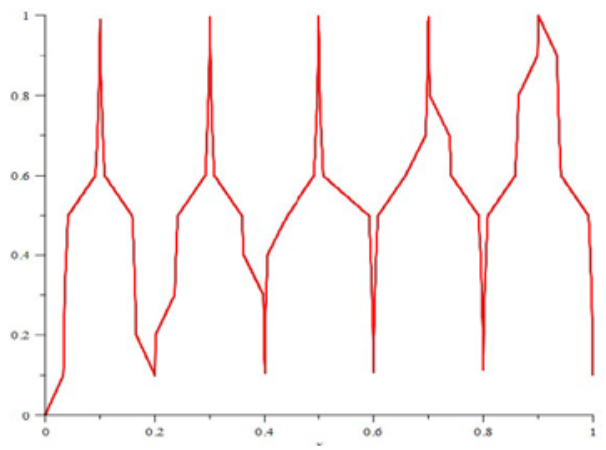

Figure 5. Semi-Markov map g preserves density fB. 


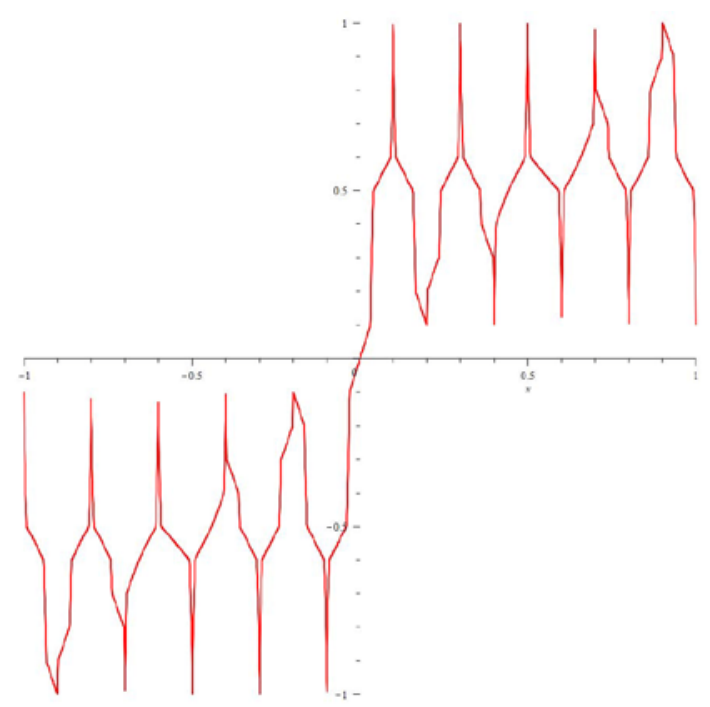

Figure 6. Map $T$ preserves two densities: $\mathrm{fB}$ and symmetric $\mathrm{fA}$.

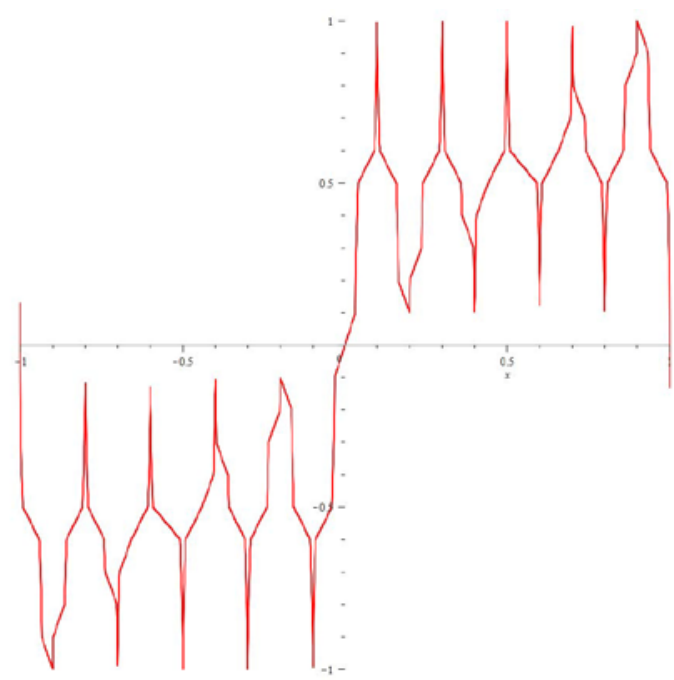

Figure 7. Metastable map $T_{\varepsilon}$ preserves unique density $f_{\varepsilon}$ which is very close to (1/2)fA + (1/2)fB.

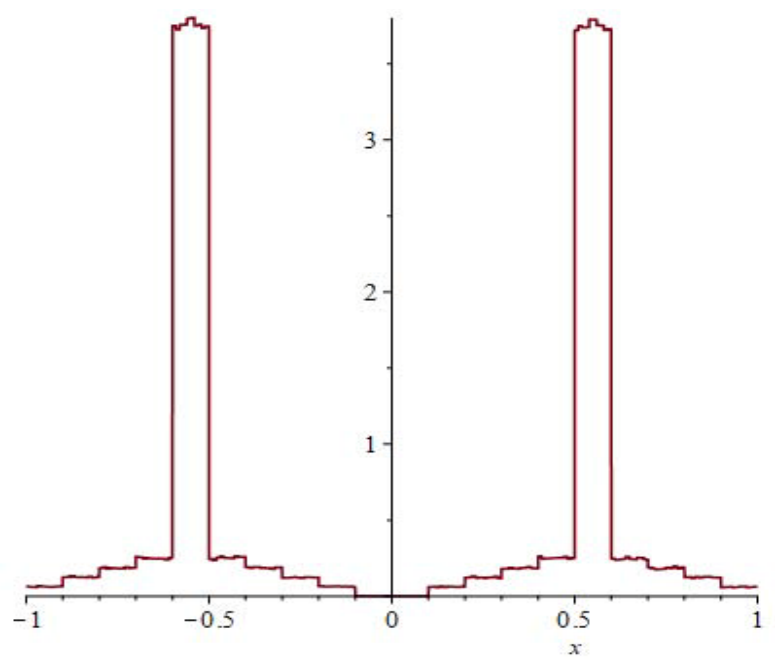

Figure 8. The computer simulation of the density $f_{\varepsilon} \approx(1 / 2) f A+(1 / 2) f B$. 


\section{Acknowledgements}

The research of the authors was supported by NSERC grants. The research of Z. Li is also supported by NNSF of China (No. 11161020 and No. 11361023).

\section{References}

[1] Tim Folger. (2018). Crossing the quantum divide, Scientific American, July 2018, 29-35.

[2] Cecilia Gonzáles-Tokman, Brian R. Hunt, and Paul Wright. (2011). Approximating invariant densities of metastable systems, Ergod. Th. \& Dynamic Sys., 31, 1345-1361.

[3] Rogers, Alan, Shorten, Robert, Heffernan, Daniel M. (2008). A novel matrix approach for controlling the invariant densities of chaotic maps, Chaos Solitons Fractals, 35(2008), no. 1, 161-175.

[4] Góra, P., Boyarsky, A. (1993). A matrix solution to the inverse Perron-Frobenius problem. Proc. Amer. Math. Soc. 118(1993), no. 2, 409-414.

[5] Boyarsky, Abraham, Góra, Pawel. (1997). Laws of chaos. Invariant measures and dynamical systems in one dimension, Probability and its Applications. Birkhäuser Boston, Inc., Boston, MA,

[6] Lasota, Andrzej, Mackey, Michael C. (1994). Chaos, fractals, and noise. Stochastic aspects of dynamics , Second edition. Applied Mathematical Sciences, 97. Springer-Verlag, New York. 